Proceeding Paper

\title{
Fluorescent Chitosan Nanogels Developed for Targeting Endothelial Cells of Axillary Lymph Nodes ${ }^{\dagger}$
}

\author{
Miruna-Silvia Stan 1,**, Ionela Cristina Nica 1, Juliette Moreau 2, Maïté Callewaert ', Cyril Cadiou 2, \\ Françoise Chuburu ${ }^{2}$, Hildegard Herman ${ }^{3}$, Anca Hermenean ${ }^{3}$, Anca Dinischiotu ${ }^{1}$ and Sorina N. Voicu ${ }^{1}$
}

Citation: Stan, M.S.; Nica, I.C.;

Moreau, J.; Callewaert, M.; Cadiou,

C.; Chuburu, F.; Herman, H.;

Hermenean, A.; Dinischiotu, A.;

Voicu, S.N. Fluorescent Chitosan

Nanogels Developed for Targeting

Endothelial Cells of Axillary Lymph

Nodes. Mater. Proc. 2021, 4, 12.

https://doi.org/10.3390/IOCN2020-

07847

Academic Editors: Ana María

Díez-Pascual, Antonio Di

Bartolomeo and Guanying Chen

Published: 11 November 2020

Publisher's Note: MDPI stays neutral with regard to jurisdictional claims in published maps and institutional affiliations.

Copyright: (C) 2020 by the authors. Submitted for possible open access publication under the terms and conditions of the Creative Commons Attribution (CC BY) license (http://creativecommons.org/licenses /by/4.0/).
1 Department of Biochemistry and Molecular Biology, Faculty of Biology, University of Bucharest, 91-95 Splaiul Independentei, 050095 Bucharest, Romania; cristina.nica@drd.unibuc.ro (I.C.N.); anca.dinischiotu@bio.unibuc.ro (A.D.); sorina.voicu@bio.unibuc.ro (S.N.V.)

2 Institute of Molecular Chemistry of Reims, UMR CNRS 7312, University of Reims Champagne Ardenne, 51100 Reims, France; juliette.moreau@univ-reims.fr (J.M.); maite.callewaert@univ-reims.fr (M.C.); cyril.cadiou@univ-reims.fr (C.C.); francoise.chuburu@univ-reims.fr (F.C.)

3 Institute of Life Sciences, Vasile Goldis Western University of Arad, 86 Rebreanu, 310414 Arad, Romania; hildegard.i.herman@gmail.com (H.H.); anca.hermenean@gmail.com (A.H.)

* Correspondence: miruna.stan@bio.unibuc.ro

+ Presented at the 2nd International Online-Conference on Nanomaterials, 15-30 November 2020; Available online: https://iocn2020.sciforum.net/.

\begin{abstract}
Nanogels are a novel class of three-dimensional cross-linked polymers able to retain high amounts of water in their network structure, with large potential applications in nanomedicine. In our study, the polymer matrix selected was chitosan, as this polysaccharide biopolymer composed of $\mathrm{N}$-acetylglucosamine and glucosamine residues exhibits great biocompatibility and low toxicity. The preparation was performed by ionic gelation in the presence of hyaluronic acid and sodium tripolyphosphate, with rhodamine or fluorescein isothiocyanate molecules grafted on a chitosan backbone. In order to validate the possible usage of these chitosan-fluorophores conjugates for fluorescence imaging purposes in cancer diagnostics and therapy, their biological effect was assessed on SVEC4-10 cells (a simian virus 40-transformed mouse microvascular endothelial cell line). Cell viability, membrane integrity and nanogels uptake were examined following exposure for 6 and 24 $\mathrm{h}$ at concentrations up to $120 \mu \mathrm{g} / \mathrm{mL}$. A good biocompatibility was obtained after both time intervals of incubation with nanogels, with no increase in cell death or membrane damage being noticed as compared to control. By examination on confocal laser scanning microscopy, both types of fluorescent nanogels agglomerated on the surface of the cell membrane, their cellular internalization being observed only for few cells, preferentially at the cell periphery. In conclusion, based on the biocompatibility of the nanogels, these can further incorporate gadolinium for an improved magnetic resonance imaging effect in nanomedicine.
\end{abstract}

Keywords: chitosan; nanoparticles; lymph nodes; medical imaging

\section{Introduction}

Nanogels are a novel class of three-dimensional cross-linked polymers able to retain high amounts of water in their network structure, with large potential applications in nanomedicine. In our study, the polymer matrix selected was chitosan, as this polysaccharide biopolymer composed of $\mathrm{N}$-acetylglucosamine and glucosamine residues exhibits great biocompatibility and low toxicity. 


\section{Materials and Methods}

The preparation was performed by ionic gelation in the presence of hyaluronic acid and sodium tripolyphosphate, with rhodamine or fluorescein isothiocyanate molecules grafted on a chitosan backbone. In order to validate the possible usage of these chitosanfluorophores conjugates for fluorescence imaging purposes in cancer diagnostics and therapy, their biological effect was assessed on SVEC4-10 cells (a simian virus 40-transformed mouse microvascular endothelial cell line). Cell viability, membrane integrity and nanogels uptake were examined following exposure for 6 and $24 \mathrm{~h}$ at concentrations up to $120 \mu \mathrm{g} / \mathrm{mL}$.

\section{Results}

A good biocompatibility was obtained after both time intervals of incubation with nanogels, with no increase in cell death or membrane damage being noticed as compared to control. By examination on confocal laser scanning microscopy, both types of fluorescent nanogels agglomerated on the surface of the cell membrane (Figure 1), their cellular internalization being observed only for few cells, preferentially at the cell periphery (Figure 2).
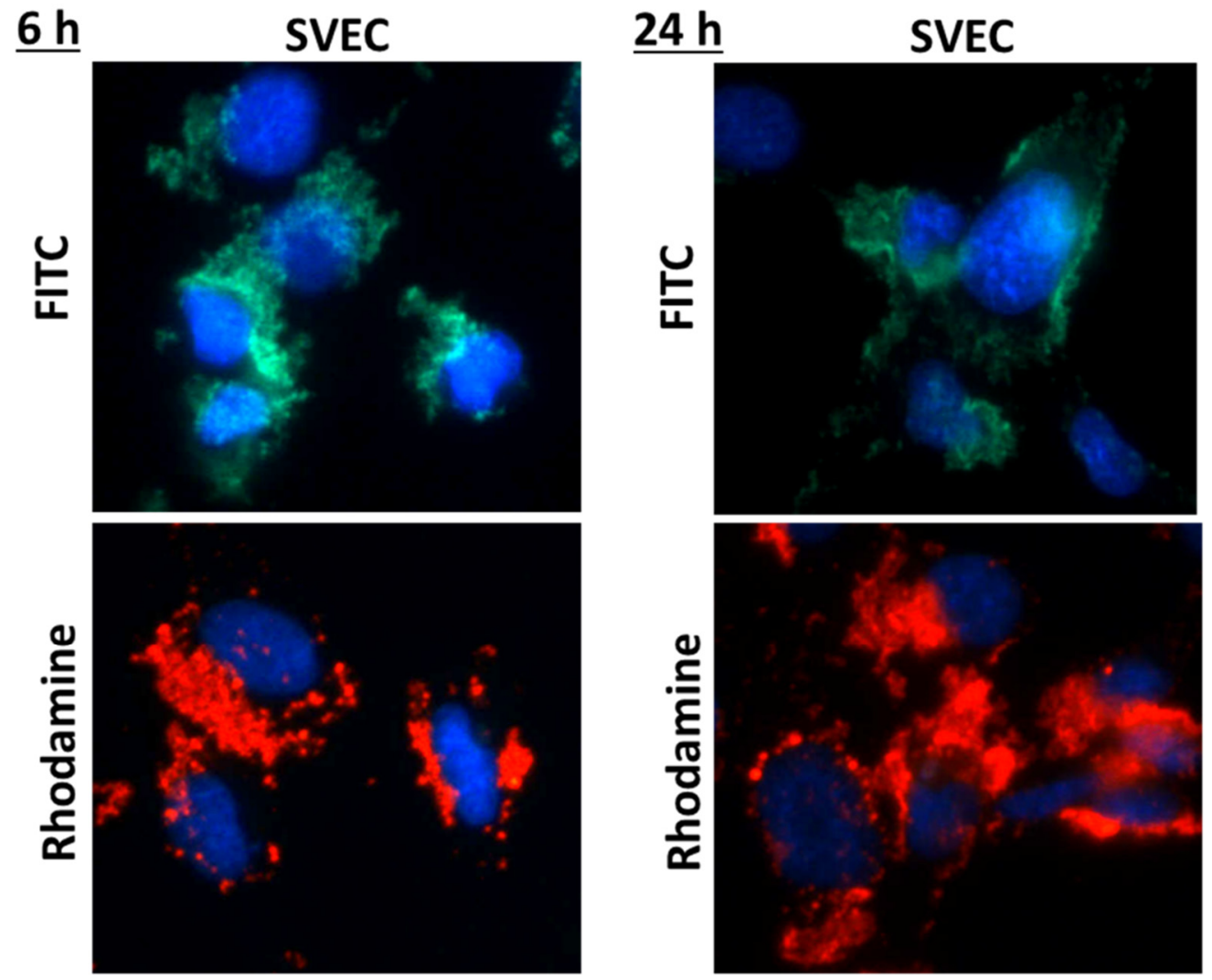

Figure 1. Fluorescence images showing the nuclei of SVEC4-10 cells stained in blue with DAPI solution and the nanogels with rhodamine or fluorescein isothiocyanate (FITC) molecules grafted on a chitosan backbone. 


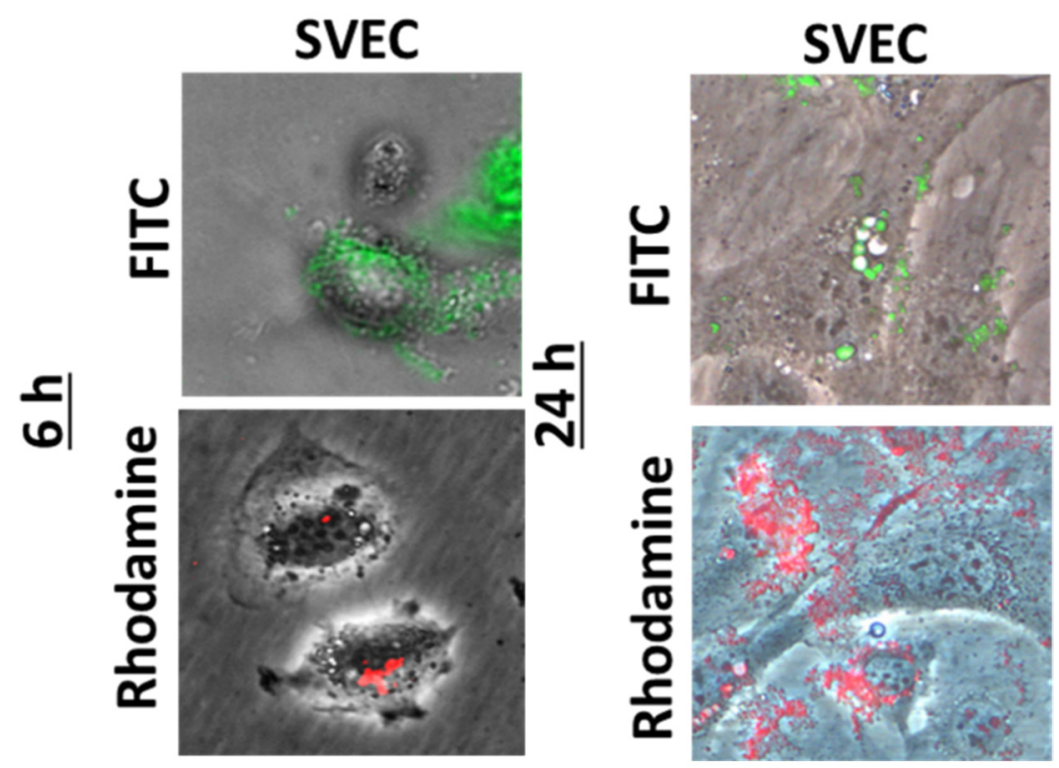

Figure 2. Fluorescence images showing the SVEC4-10 cells in phase contrast and the nanogels with rhodamine (red) or fluorescein isothiocyanate (FITC, green) molecules grafted on a chitosan backbone.

\section{Conclusions}

Based on the biocompatibility of the nanogels, these can further incorporate gadolinium for an improved magnetic resonance imaging effect in nanomedicine.

Funding: This work was supported by a grant of the Romanian Ministry of Research and Innovation, CCCDI-UEFISCDI, project number PN-III-P3-3.1-PM-RO-FR-2019-0204/6BM/2019, within PNCDI III.

Institutional Review Board Statement: Not applicable.

Informed Consent Statement: Not applicable.

Data Availability Statement: The data is available at the request from the corresponding author.

Conflicts of Interest: The authors declare no conflict of interest. The funders had no role in the design of the study; in the collection, analyses, or interpretation of data; in the writing of the manuscript, or in the decision to publish the results. 